\title{
Radiation Pattern Performance of Unequally Linear Arrays with Parasitic Element
}

\author{
Noor Ainniesafina Zainal ${ }^{1}$, Muhammad Ramlee Kamarudin ${ }^{\star^{2}}$, Yoshihide Yamada ${ }^{3}$, \\ Norhudah Seman ${ }^{4}$ \\ ${ }^{1}$ Department of Polytechnic, Ministry of Education Malaysia, Presint 4, 62100 Wilayah Persekutuan \\ Putrajaya, Malaysia \\ ${ }^{1,2,4}$ Wireless Communication Center (WCC), Universiti Teknologi Malaysia (UTM), 81310 Skudai, Johor, \\ Malaysia \\ ${ }^{3}$ Malaysia-Japan International Institute of Technology (MJIIT), Universiti Teknologi Malaysia, 54100 K. L, \\ Malaysia \\ ${ }^{*}$ Corresponding author, e-mail: ramlee@fke.utm.my
}

\begin{abstract}
For next generation of $5 G$ mobile base station antennas, multibeam, multifrequency and low sidelobe characteristics requested. Simplify the feeding network will contribute a low feeder loss and frequency dependent. From the previous research by the author, low sidelobe level reported by density tapered array configuration from $-13 d B$ to $-16 d B$ and the result maintained for wideband operation frequency at $28 \mathrm{GHz}, 42 \mathrm{GHz}$, and $56 \mathrm{GHz}$. However, the grating lobe has occurred due to element spacing larger than a wavelength of higher frequency $(56 \mathrm{GHz})$. In this paper, an investigation was made of the performance of radiation pattern for unequally microstrip linear array antenna in frequency $42 \mathrm{GHz}$ and $56 \mathrm{GHz}$ by loading parasitic elements. The effect of parasitic element to the impedance, gain, and sidelobe level of unequally microstrip linear spaced tapered array also examined. The design has been simulated using Ansoft High Frequency Structural Simulator (HFSS) ver 16.0.
\end{abstract}

Keywords: 5G, unequally microstrip linear array, sidelobe, grating lobe, parasitic element

Copyright $\odot 2017$ Institute of Advanced Engineering and Science. All rights reserved.

\section{Introduction}

The fifth generation $(5 \mathrm{G})$ wireless communication technology will be implemented in the coming year and providing great broadcasting of data in Gbps to support a dense data traffic. Therefore, millimeter wave communication systems are required as to support the technology requirements. The trend of mobile communication system has led to the development of incipient technologies with multiband capabilities. The future $5 \mathrm{G}$ mobile base station antenna technology needs the multibeam and multifrequency antenna with low sidelobe level to reduce the interference to other frequency reuse cell [1]. For achieving low sidelobe over wide frequency characteristic, a density tapered array configuration is promising [2]. For this purpose, the array excitation coefficient has a same amplitude and phase in all array elements due to the same length of feeding and contributes no frequency dependence. Therefore, the low sidelobe level over wide frequency range expected.

In array antennas design, the element spacing of array is important and requires being within one wavelength. However, the density tapered array antenna configurations over wide frequency band have element spacing larger than wavelength especially at high frequencies [2]. Thus, it will generate large grating lobes similar to the main lobe and also decrease the gain of the main lobe because of the radiation from unnecessary lobes [3]. The problem of grating lobe is not too noticeable for millimeter wave due to the attenuation in the atmosphere is large [4]. However, reduce the grating lobe will increase the performance of the entire array antenna. Few attempts on grating lobe reduction have been made in previous with microstrip patch aperiodic array at $24 \mathrm{GHz}$ [5] based on geometric tapered distribution and [6] introduce the genetic algorithm in aperiodic arrays configuration for grating lobe suppression. Besides, a new design concept of subarrays with a parasitic element for equal array has developed by [7] whereby the 
parasitic elements have the effects on the radiation pattern [8]. However these parasitic loading techniques applied so far to the uniform array.

We hereby propose the concept of loading parasitic elements between the 16 array radiating elements to identify the changes of the radiation pattern of unequally linear array antenna. In this paper, practical data by loading parasitic elements on wide band characteristics investigated through calculation results by using a High Frequency Structure Simulator (HFSS). The dimension size of a parasitic patch is parameters in this study. Relation of grating lobe suppression and unequally spaced linear array obtained and the gain increased are expected especially in the high frequency due to grating lobe reduction.

\section{Research Method}

Grating lobe occurs in uniformly spaced array when the antenna element separation is too large (greater than one wavelength). The angle where grating lobe start form is given by the next expression (1).

$$
\theta_{G L}=\sin ^{-1}\left( \pm n \frac{\lambda_{o}}{d}\right)
$$

The main beam with $n=0$ gives no grating lobe. In this case, grating lobe started at $47.4^{\circ}$ degree angle. Based on the previous research by the author, low sidelobe level characteristics over wide frequency range would be achieved by a density tapered array configurations [2]. In this paper, three concepts of the array with different element spacing $(d)$ and electric field density presented. The array configurations consist of Equally Spaced Array (ESA) with $d_{c}=$ $0.7 \lambda_{o}$, Density Tapered Array 1 (DTA 1) with $d_{c}=0.6 \lambda_{o}$ and Density Tapered Array 2 (DTA 2) with $d_{c}=0.5 \lambda_{o}$. Figure $1(\mathrm{a})$ shows the configuration of 16 elements of the antenna for Equally Spaced Array (ESA). In the case of DTA 1 and DTA 2, the distance between the center and the other elements is not the same and increased at the end of the array as shown in Figure 1(b).

The 16 patch elements are excited by the standard coaxial feed arrangement with $50 \Omega$ input impedance. Three dimension of antenna are $\mathrm{W}=\mathrm{L}=3.27 \mathrm{~mm}, \mathrm{~W}=\mathrm{L}=2.14 \mathrm{~mm}$ and $\mathrm{W}=$ $\mathrm{L}=1.64 \mathrm{~mm}$ which are designed to operate in frequency $f_{0}=28 \mathrm{GHz}, f_{1}=42 \mathrm{GHz}$ and $f_{2}=56$ $\mathrm{GHz}$ respectively. In the case of unequally spaced linear array, the lower frequency $(28 \mathrm{GHz})$ with the wavelength $\left(\lambda_{0}\right)$ is used as a reference. All three configurations of the array designed onto Rogers RT/Duroid $5880\left(\varepsilon_{r}=2.2\right.$ and $\left.\tan \delta=0.0009\right)$ with thickness of $0.508 \mathrm{~mm}$ and array dimension of $15 \mathrm{~mm} \times 130 \mathrm{~mm} \times 0.508 \mathrm{~mm}^{3}$ (Length $\times$ Width $\times$ Height). The length of all array fixed with $L=15 \times 0.7 \lambda_{o}(=112.5 \mathrm{~mm})$. Meanwhile, the author has reported the length at high frequency $(56 \mathrm{GHz})$ is greater than one wavelength [2].

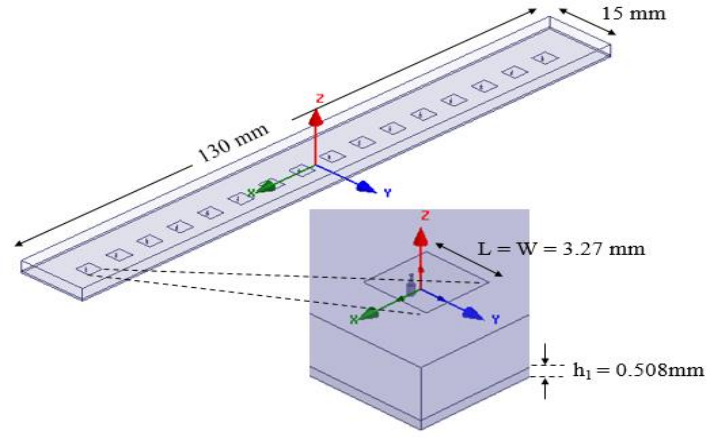

(a)

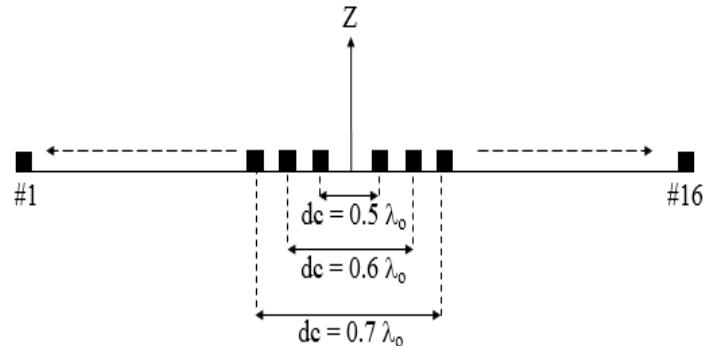

(b)

Figure 1. Antenna Array configurations. (a) 3D view of Equally Spaced Array (ESA, $d_{c}=0.7 \lambda_{o}$ configuration at $28 \mathrm{GHz}$. (b) Antenna array with different center of element spacing, ESA ( $d_{c}=$ $\left.0.7 \lambda_{o}\right)$, DTA $1\left(d_{c}=0.6 \lambda_{o}\right)$ and DTA $2\left(d_{c}=0.5 \lambda_{o}\right)$. 
Array antenna with nonuniform or unequally spaced array allows controlling the radiation pattern. Sidelobe level reduced by density tapered array configuration. Figue 2 shows the characteristics of low sidelobe over a wide frequency band. The grating lobe is clear occur at frequency $f_{2}=56 \mathrm{GHz}$ while the lobes far from the beam are increased appreciably at frequency operate $f_{1}=42 \mathrm{GHz}$.

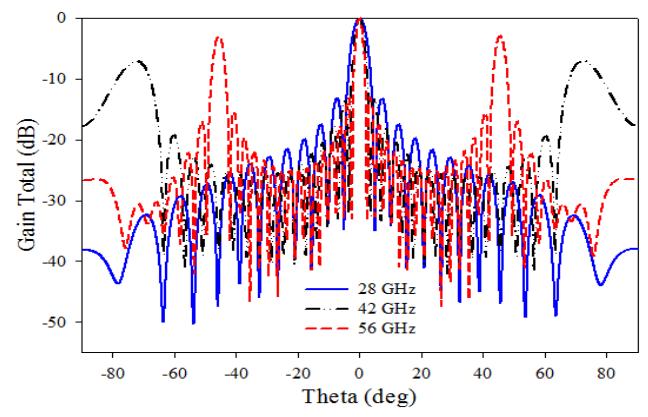

Figure 2. The performance of grating lobe over wide bandwidth characteristics for Equally Spaced Array (ESA) configurations [2]

In this letter, parasitic element is arranged to be placed at the center in between of radiating patch and excited by the electromagnetic coupling. Two dimension size of the parasitic element is studied as shown in Table 1. The radiation pattern designed in the vertical plane as the $\mathrm{x}-\mathrm{z}$ plane. Fig. 3 shows the equally spaced linear array antenna at $42 \mathrm{GHz}$ without and with a different dimension size of parasitic element configurations for comparing their performance in term of bandwidth, gain and radiation characteristics.

Table 1. Parameter dimension of patch array antenna and parasitic element

\begin{tabular}{cccccccc}
\hline Parameter & \multicolumn{3}{c}{ Patch antenna } & \multicolumn{4}{c}{ Parasitic element } \\
\cline { 2 - 7 } & $28 \mathrm{GHz}$ & $42 \mathrm{GHz}$ & $56 \mathrm{GHz}$ & \multicolumn{3}{c}{$42 \mathrm{GHz}$} & \multicolumn{3}{c}{$56 \mathrm{GHz}$} \\
\cline { 4 - 7 } & & & & Design 1 & Design 2 & Design 1 & Design 2 \\
\hline $\mathrm{W}(\mathrm{mm})$ & 3.27 & 2.14 & 1.64 & 0.3 & 2.14 & 0.3 & 1.64 \\
$\mathrm{~L}(\mathrm{~mm})$ & 3.27 & 2.14 & 1.64 & 2.14 & 2.14 & 1.64 & 1.64 \\
\hline
\end{tabular}

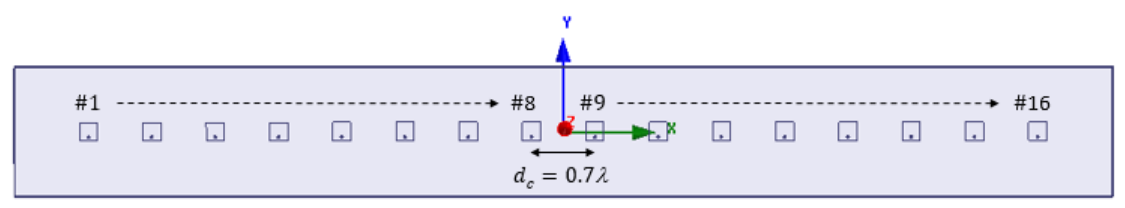

(a)

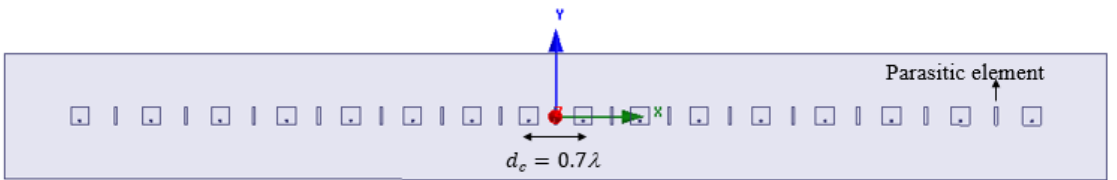

(b)

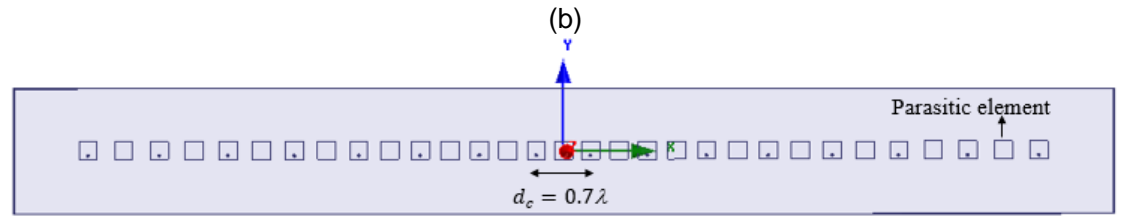

(c)

Figure 3. Simulated design. (a) ESA without parasitic. (b) ESA with parasitic Design 1. (c) ESA with parasitic Design 2 


\section{Simulation Results and Analysis}

In the calculation, High Frequency Structure Simulator (HFSS) employed. Fig. 4 (a) shows the gain for $42 \mathrm{GHz}$ decreased from $19.29 \mathrm{dBi}$ to $18.40 \mathrm{dBi}$ for ESA because of the sidelobe far from the main lobe are increased. From the previous literature, parasitic elements will increase the gain and bandwidth. However, the result was not in expected because of the concept of a multifrequency antenna with the unequally spaced array that has described previously. Meanwhile, in high frequency Fig. 4 (b) shows the gain for $56 \mathrm{GHz}$ (ESA) increased with a parasitic element from $19.12 \mathrm{dBi}$ to $20.31 \mathrm{dBi}$ due to a decrease of grating peaks. The gain for DTA 1 and DTA 2 also increased but lower than ESA because the spacing between elements is nonuniform and electromagnetic coupling become lower toward the edge of the array.

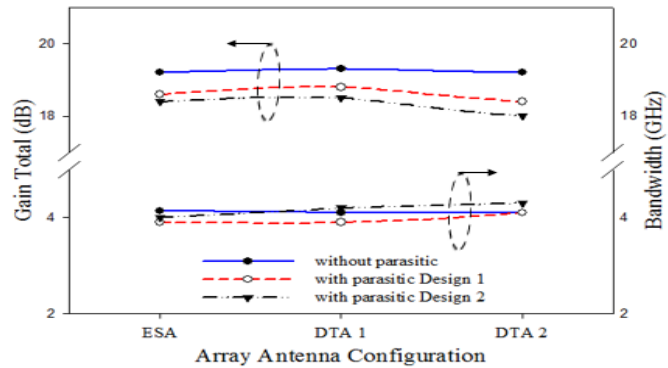

(a)

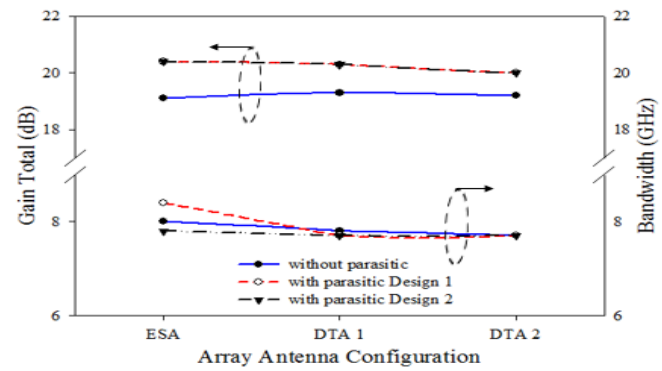

(b)

Figure 4. Gain vs. Bandwidth. (a) $42 \mathrm{GHz}$. (b) $56 \mathrm{GHz}$

The grating lobe characteristics are only focusing on frequency $56 \mathrm{GHz}$ because no grating lobe appeared in a lower frequency (refer Figue 2). Figure 5(a) shows 2D ESA radiation pattern where the grating lobe slightly reduced from $-2.9 \mathrm{dBi}$ to $-6.0 \mathrm{dBi}$. DTA 1 and DTA 2 also shows the reduction with $\Delta=2.8 \mathrm{dBi}$ and $\Delta=2.7 \mathrm{dBi}$ respectively. Meanwhile, the sidelobe far from the main beam also slightly decreased as shown in Figure 5 (b)-(c). In lower frequency, Figure 5 (d) shows the lowest sidelobe achieved for DTA 2 configuration with parasitic Design 1 from $-19.35 \mathrm{dBi}$ to $-26.19 \mathrm{dBi}$.

Table 2. Summary of performances of three configurations of array without and with parasitic elements

\begin{tabular}{|c|c|c|c|c|c|c|c|c|c|c|}
\hline \multirow[t]{3}{*}{ Parameter } & \multirow{3}{*}{$\begin{array}{c}\text { Frequency } \\
(\mathrm{GHZ})\end{array}$} & \multicolumn{8}{|c|}{ Array configuration } & \\
\hline & & \multicolumn{3}{|c|}{ ESA } & \multicolumn{3}{|c|}{ DTA 1} & \multicolumn{3}{|c|}{ DTA 2} \\
\hline & & WP & WP 1 & $\begin{array}{c}\text { WPD } \\
2\end{array}$ & WP & $\begin{array}{c}\text { WPD } \\
1\end{array}$ & $\begin{array}{c}\text { WPD } \\
2\end{array}$ & WP & $\begin{array}{c}\text { WPD } \\
1\end{array}$ & $\begin{array}{c}\text { WPD } \\
2\end{array}$ \\
\hline \multirow{2}{*}{$\mathrm{S}_{11}(\mathrm{~dB})$} & 42 & 41.96 & 42.07 & 41.78 & 41.94 & 42.07 & 41.87 & 41.94 & 42.00 & 42.00 \\
\hline & 56 & 56.01 & 56.04 & 56.04 & 56.04 & 56.10 & 56.06 & 56.04 & 56.04 & 56.06 \\
\hline \multirow{2}{*}{$\begin{array}{l}\text { Bandwidth } \\
(\mathrm{GHz})\end{array}$} & 42 & 4.14 & 3.85 & 3.99 & 4.13 & 3.93 & 4.21 & 4.12 & 4.10 & 4.26 \\
\hline & 56 & 8.04 & 8.36 & 7.81 & 7.75 & 7.77 & 7.72 & 7.77 & 7.66 & 7.72 \\
\hline \multirow{2}{*}{ Gain $(\mathrm{dBi})$} & 42 & 19.29 & 18.62 & 18.40 & 19.35 & 18.75 & 18.49 & 19.25 & 18.44 & 17.85 \\
\hline & 56 & 19.12 & 20.42 & 20.31 & 19.35 & 20.33 & 20.26 & 19.29 & 19.95 & 19.73 \\
\hline$-3 d B$ & 42 & 3.02 & 3.01 & 3.04 & 3.22 & 3.15 & 3.00 & 3.29 & 3.32 & 2.84 \\
\hline HPBW & 56 & 2.21 & 2.23 & 2.22 & 2.36 & 2.32 & 2.30 & 2.46 & 2.36 & 2.36 \\
\hline \multirow{2}{*}{ SLL (dB) } & 42 & -13.23 & -13.34 & -13.35 & -16.49 & -18.89 & -13.68 & -19.35 & -26.19 & -8.79 \\
\hline & 56 & -13.23 & -14.07 & -13.55 & -16.14 & -15.66 & -15.52 & -19.86 & -14.16 & -15.32 \\
\hline Grating & 42 & - & - & - & - & - & - & - & - & - \\
\hline Lobe (dB) & 56 & -2.90 & -6.03 & -5.55 & -6.97 & -9.75 & -9.70 & -8.44 & -11.12 & -10.93 \\
\hline
\end{tabular}

Table 2 shows the summary of the performance for three array configurations. The impedance of each antenna is almost the same. The antenna in frequency $56 \mathrm{GHz}$ achieved the highest gain compared to the lower frequency antenna due to the reduction of peaks lobe. In the simulation, it demonstrated that the gain improved by $1.3 \mathrm{dBi}$ by loading 15 parasitic elements. It indicates that the near field coupling effects on the parasitic element to become a radiating elements in an array. The parasitic element clearly enhances the bandwidth for the array configuration with Design 2 due to the stronger interaction between non-radiating edges 
[9] and the coupling is strong enough with small spacing between elements. Half power bandwidth is almost the same for all array configurations. As a result, the smaller dimension size (Design 1) of parasitic element produce optimal coupling characteristics to increase the gain and slightly reduce the grating lobe.

Table 2 shows the summary of the performance for three array configurations. The impedance of each antenna is almost the same. The antenna in frequency $56 \mathrm{GHz}$ achieved the highest gain compared to the lower frequency antenna due to the reduction of peaks lobe. In the simulation, it demonstrated that the gain improved by $1.3 \mathrm{dBi}$ by loading 15 parasitic elements. It indicates that the near field coupling effects on the parasitic element to become a radiating elements in an array. The parasitic element clearly enhances the bandwidth for the array configuration with Design 2 due to the stronger interaction between non-radiating edges [9] and the coupling is strong enough with small spacing between elements. Half power bandwidth is almost the same for all array configurations. As a result, the smaller dimension size (Design 1) of parasitic element produce optimal coupling characteristics to increase the gain and slightly reduce the grating lobe.
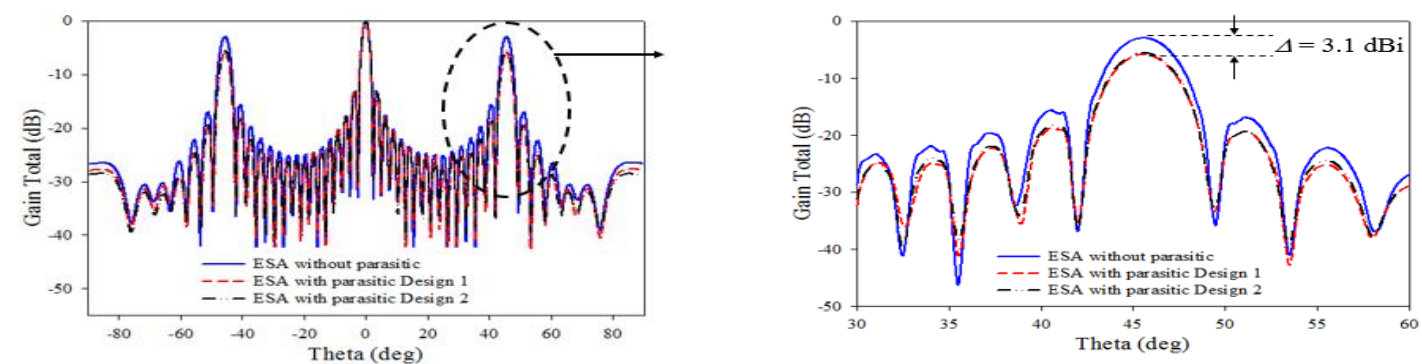

(a)

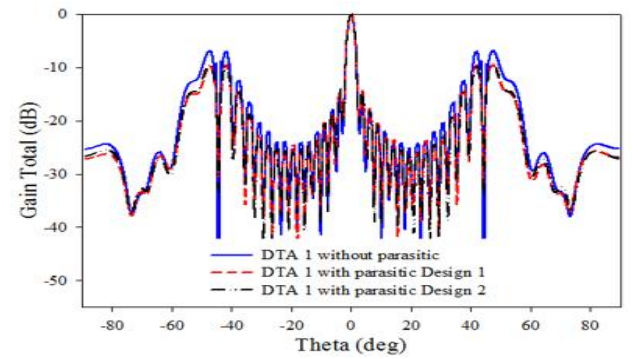

(b)
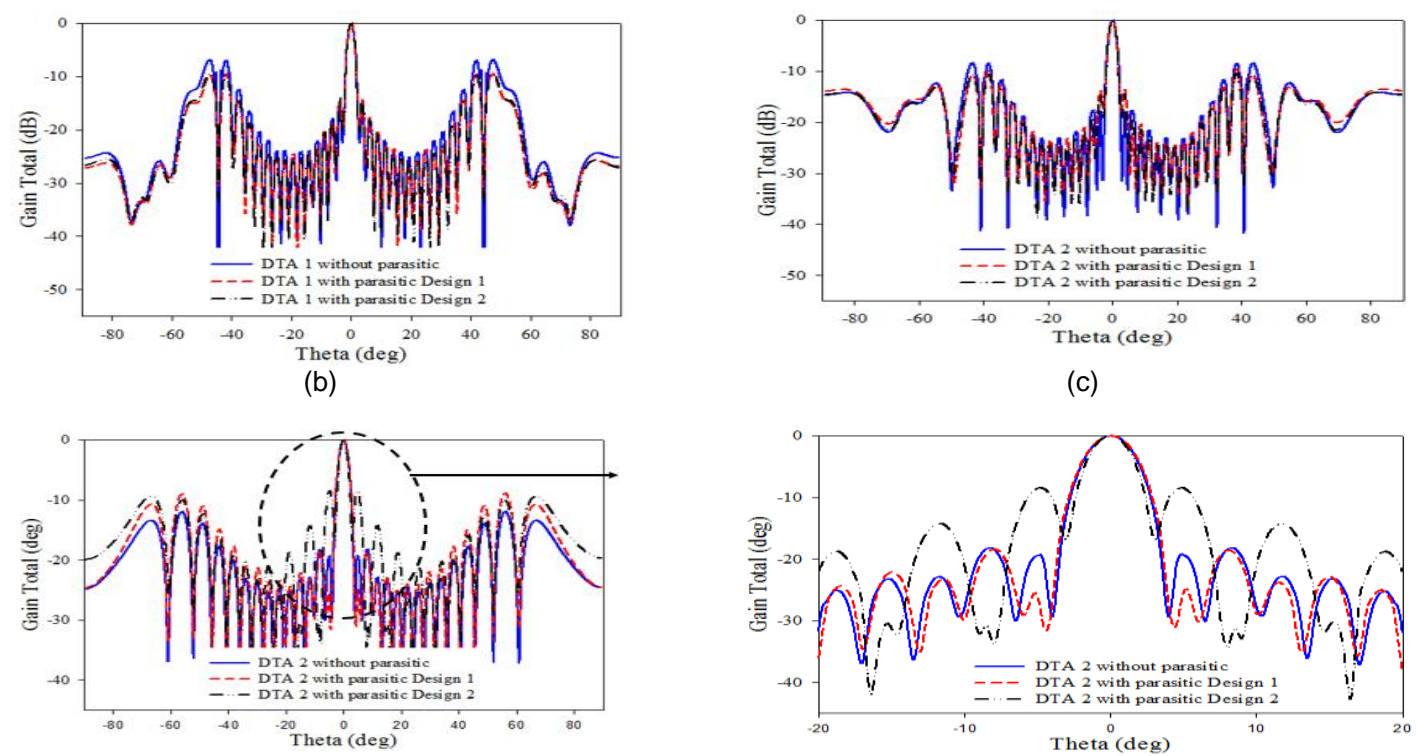

(c)

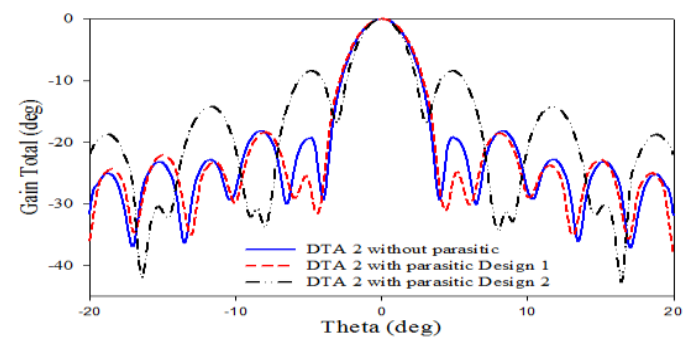

(d)

Figure 5. The characteristics of radiation pattern, (a)-(c) Grating lobe characteristic in frequency $56 \mathrm{GHz}$ (all array configuration). (d) Sidelobe characteristics in frequency $42 \mathrm{GHz}$ (DTA 2)

\section{Conclusion}

In this letter, the effects of parasitic elements to the characteristics of grating lobe for unequally linear array antennas were simulated and analyzed. The gain increased as expected in high frequency due to grating lobe reduction and better sidelobe level in a lower frequency. The gain and radiation performance of the proposed array antenna are depended strongly on the near field coupling of the parasitic element by obtaining energies from the radiating fed 
elements. By using a small dimension size of the parasitic element gives a better performance of the radiation characteristics of unequally spaced linear array antenna.

\section{References}

[1] M. Kijima, et al. Developemet of Dual-Frequency Base Station Antenna for Cellular Mobile Radios. IEICE Transaction on Communication. 1999; E82-B(4): 636-644.

[2] N. A. Zainal, et al. Low Sidelobe and Widebad Characteristics of Density Tapered Arrays for 5G Mobile Systems. Jurnal Teknologi. 2016; 7(6-2): 71-76.

[3] C. A. Balanis, "Antenna theory Analysis and Design," $3^{\text {rd }}$. ed., Wiley, 2005, pp. 297.

[4] A. Vosoogh, et al. Simple Formula for Aperture Efficiency Reduction Due to Grating Lobes in Planar Phased Arrays. IEEE Transaction Antennas Propagation. 2016; 64(6): 2263-2269.

[5] S. Su'arez, et al. Experimental Validation of Linear Aperiodic Array for Grating Lobe Suppression. Progress In Electromagnetics Research. 2012; 26: 193-203.

[6] W. C. Barott, et al. Grating Lobe Reduction in Aperiodic Linear Arrays of Physically Large Antennas. IEEE Antennas and Wireless Propagation Letters. 2009; 8: 406-408.

[7] T. Suda, et al., "Grating Lobe Suppression in an Array Antenna with Element Spacing Greater Than a Half Wavelength," in Antennas and Propagation Society International Symposium (APSURSI), 2010 IEEE, 2010.

[8] R. Q. LEE, et al. Radiation Characteristics of Microstrip Arrays with Parasitic Elements. Electronics Letters. 1987; 23(16): 835-837.

[9] R. Q. Lee, et al., "An Experimental Investigation of Parasitic Microstrip Arrays," in Symposium on Antenna Application, Sept 23-25, 1987. 